\title{
Bone Formation with Deproteinized Bovine Bone Mineral or Biphasic Calcium Phosphate in the Presence of Autologous Platelet Lysate: Comparative Investigation in Rabbit
}

\author{
Carole Chakar, ${ }^{1,2}$ Nada Naaman, ${ }^{1}$ Emmanuel Soffer, ${ }^{2,3}$ Nicolas Cohen,, \\ Nada El Osta, ${ }^{5}$ Hervé Petite, ${ }^{2}$ and Fani Anagnostou ${ }^{2,4}$ \\ ${ }^{1}$ Department of Periodontology, School of Dentistry, Saint-Joseph University, P.O. Box 11-5076, Riad el Solh, Beirut 1107 2050, Lebanon \\ ${ }^{2}$ Laboratoire de Bioingénierie et Biomécanique Ostéo-Articulaires, UMR CNRS 7052, PRES Sorbonne Paris Cité, 75010 Paris, France \\ ${ }^{3}$ Department of Prosthetic Dentistry, Service of Odontology, Pitié-Salpêtrière Hospital, AP-HP, U.F.R. d'Odontologie, \\ Paris 7-Diderot University, Paris, France \\ ${ }^{4}$ Department of Periodontology, Service of Odontology, Pitié-Salpêtrière Hospital, AP-HP, U.F.R. d'Odontologie, \\ Paris 7-Diderot University, Paris, France \\ ${ }^{5}$ Department of Prosthetic Dentistry, School of Dentistry, Saint-Joseph University, P.O. Box 11-5076, Riad el Solh, \\ Beirut 1107 2050, Lebanon
}

Correspondence should be addressed to Fani Anagnostou; fani.anagnostou@univ-paris-diderot.fr

Received 11 January 2014; Revised 8 May 2014; Accepted 9 May 2014; Published 27 May 2014

Academic Editor: Fen-Huei Lin

Copyright (C) 2014 Carole Chakar et al. This is an open access article distributed under the Creative Commons Attribution License, which permits unrestricted use, distribution, and reproduction in any medium, provided the original work is properly cited.

\begin{abstract}
Bone substitutes alone or supplemented with platelet-derived concentrates are widely used to promote bone regeneration but their potency remains controversial. The aim of this study was, therefore, to compare the regenerative potential of preparations containing autologous platelet lysate (APL) and particles of either deproteinized bovine bone mineral (DBBM) or biphasic calcium phosphate (BCP), two bone substitutes with different resorption patterns. Rabbit APL was prepared by freeze-thawing a platelet suspension. Critical-size defects in rabbit femoral condyle were filled with DBBM or DBBM+APL and BCP or BCP+APL. Rabbits were sacrificed after six weeks and newly formed bone and residual implanted material were evaluated using nondemineralized histology and histomorphometry. New bone was observed around particles of all fillers tested. In the defects filled with BCP, the newly formed bone area was greater $(70 \% ; P<0.001)$ while the residual material area was lower $(60 \% ; P<0.001)$ than that observed in those filled with DBBM. New bone and residual material area of defects filled with either APL+DBBM or APL+BCP were similar to those observed in those filled with the material alone. In summary, osteoconductivity and resorption of BCP were greater than those of DBBM, while APL associated with either DBBM or BCP did not have an additional benefit.
\end{abstract}

\section{Introduction}

Various bone-substitute materials are used to promote bone reconstruction in oral and maxillofacial areas. They have osteoconductivity properties, as they provide a scaffold for cell adhesion and proliferation and allow bone growth on their surfaces. Deproteinized bovine bone matrix (DBBM) and biphasic calcium phosphate (BCP) are two calcium phosphate-based bone substitutes with different resorption patterns, widely used in the management of periodontal and peri-implant bone defects as well as of bone augmentation procedures [1-4]. DBBM is a bone substitute of natural origin. It is a bovine bone that undergoes a low heat $\left(300^{\circ} \mathrm{C}\right)$ chemical extraction process by which all organic components are removed, but it maintains the natural architecture of bone and results in $\mathrm{CaP}$ crystals characterized as hydroxyapatite with morphological and structural properties similar to that of human bone hydroxyapatite [5]. In vivo, it is resorbed very slowly or not at all [6]. Tested extensively in vitro and in vivo, this material has demonstrated osteoconductive properties that improve bone regeneration [7]. BCPs are synthetic bone substitutes with different ratios of hydroxyapatite HA 
and beta-form of tricalcium phosphate ( $\beta$-TCP) depending on the preparation and synthesis procedure. Variations of BCP regarding HA/TCP ratio showed different patterns in biodegradation and osteoconduction $[8,9]$. Some clinical studies comparing BCP (60/40 HA/TCP ratio) and DBBM regenerative potential have reported conflicting results [2$4,10]$ while preclinical studies using BCP in comparison with DBBM in standardized critical-size defects are not consistent $[9,11-13]$.

To promote bone regeneration, the aforementioned bone substitutes are supplemented with autologous platelet concentrates such as platelet-rich fibrin (PRF), platelet-rich plasma (PRP) and platelet lysate (PL) [14, 15]. Activated platelets release several chemical compounds including various growth factors and cytokines that are involved in wound healing, angiogenesis, and bone regeneration processes. It is hypothesized, therefore, that a platelet concentrate delivered at the site of a bone defect may increase the local concentration of bioactive compounds, such as growth factors, and subsequently affect functions of bone-healing related cells. Despite the attractive rationale and the in vitro observed beneficial effects of platelet concentrates [16], in vivo preclinical [17-23] and clinical [14,24] studies, which evaluated the efficiency of particulate bone substitutes supplemented with platelet concentrates in the treatment of intrabony defects, have provided promising but not consistent results. With the exception of the biological environment of the defect, differences in key parameters that include implanted material behavior [25], mode of preparation of the plateletconcentrates, and/or individual variability $[18,26]$ could explain these discrepancies. In particular, most of these studies are focused on the effects of platelets concentrates in the form of PRP, a gel obtained by treating concentrated platelets with thrombin. In this case, the observed results on bone healing might be due to formation of a fibrin clot/gel, which held material particles in situ, and/or to the effects of the compounds released by the activated platelets.

Alternatively, platelet concentrates are used also in the platelet lysate form (PL). Platelet lysate is prepared from PRP by freezing platelets [27]. In vitro studies showed that PL improves the migration and/or proliferation of bone healing related cells [28-30] and is used for the clinical grade expansion of hMSC. Clinical studies suggested that it promotes oral lesions healing [31] and could be potentially useful in bone reconstructive surgery [32, 33]. However, the effect of PL in conjunction with particulate bone substitutes on bone healing has not been determined.

It is hypothesized that the mixture of bioactive molecules in the PL added to a bone substitute promotes bone healing and its effect depends on the implanted material. The objective of the present study was to compare healing around particulate DBBM and BCP and to evaluate the benefit of APL use on the potential of these bone graft substitutes to promote bone repair. To investigate these aspects, DBBM and $\mathrm{BCP}$ were associated with autologous PL and implanted in critical-size femoral defects, in rabbits.

\section{Materials and Methods}

2.1. Materials. The Materials used in the present study were as follows:

(i) particles (500-1000 microns in diameter) of biphasic calcium phosphate (BCP; BoneCeramic; Straumann, AGBasel, Switzerland), a composite of $60 \%$ hydroxyapatite (100\% crystalline), and $40 \% \beta$-Tricalcium phosphate, with $90 \%$ porosity and interconnected pores. This material is used as an alloplastic bone graft substitute in dental and orthopedic clinical practice;

(ii) particles (250-1000 $\mu \mathrm{m}$ in diameter) of deproteinized bovine bone DBBM (Bio-Oss; Geistlich, Switzerland), obtained from excised bone through a multistage purification process. The specimens had a porosity of $75-80 \%$ and interconnected pores. This material is a xenograft bone substitute.

2.2. Animals. Twelve four-month-old male New Zealand rabbits (Segav, Saint-Mars d'Egrenne, France) with a mean weight of $3.5 \mathrm{~kg}$ were used in the present in vivo study. Animal procedures were approved by the local ethics committee (Comité 4, Île-de-France, Paris, France). The animals were housed individually in metal hutches in an appropriate environment (ambient temperature of $21^{\circ} \mathrm{C}$ and $50 \%$ air humidity) that met the requirements of the European Guidelines for Care and Use of Laboratory Animals (Directive du conseil 24.11.1986, 86/609/CEE). Artificial light conditions were used in the animal facility to maintain a normal day/night biological rhythm in the animals. The animals drank water and ate commercial food concentrates (Pietrement, Sainte Colombe France) ad libitum.

2.2.1. Preparation of Rabbit APL. Rabbit APL was prepared one week before surgery and stored according to previously established methods [18]. Briefly, G21 infusion sets (Ago Microperfusore, Artsana, Como, Italy) were used to obtain $10 \mathrm{~mL}$ blood from the intermediate branch of the caudal auricular vein from each rabbit. The blood samples were collected in Venoject tubes (Centravet, Plancoet, France) containing ACD-A: citric acid-dextrose-formula A (B Braun Medical S.A., Boulogne, France) (1:3 ACD-A (v/v)/blood) and gently centrifuged at $150 \times \mathrm{g}$ and $20^{\circ} \mathrm{C}$ for $10 \mathrm{~min}$. Aliquots $(5 \mathrm{~mL})$ of the platelet-rich supernatant plasma were mixed with $0.6 \mathrm{~mL}$ ACD-A and centrifuged at $1500 \times \mathrm{g}$ and $20^{\circ} \mathrm{C}$ for $10 \mathrm{~min}$ to obtain platelet pellets. The supernatant plateletpoor plasma (PPP) was removed and stored at $-80^{\circ} \mathrm{C}$. The packed platelets were suspended in isotonic saline to obtain a platelet suspension of $10^{9}$ platelets $/ \mathrm{mL}$. Each APL was obtained using a series of five cycles of freezing-thawing of the respective autologous platelet suspensions followed by centrifugation at $10^{4} \times \mathrm{g}$ and $4^{\circ} \mathrm{C}$ for 1 hour to remove platelet membranes and other cell related debris and then stored at $-80^{\circ} \mathrm{C}$. The biological activity of APL, with different concentrations of suspended platelets $\left(0.01-0.3 \times 10^{9}\right.$ cells $\left./ \mathrm{mL}\right)$, was also assessed in vitro by testing its mitogenic effect. Preconfluent foetal rat calvaria cells were incubated with rabbit APL used in the present study or not (controls) for $24 \mathrm{~h}$. 
The cells were then removed by trypsinisation and counted using established techniques [28]. Platelet lysate enhanced, respectively, the proliferation of foetal rat calvaria cells by about $100 \%$ to $200 \%$.

2.2.2. Animal Group. The rabbits tested in the present study were randomly assigned to one of two groups, each composed of six animals. All animals underwent bilateral femoral surgery. The femoral defects in the first group of rabbits were randomly filled either with $250 \mathrm{~mm}^{3}$ DBBM mixed with $250 \mu \mathrm{L}$ of physiological saline (control) or with DBBM mixed with $250 \mu \mathrm{L}$ APL (with a platelet concentration of approximately $10^{6}$ platelets $/ \mu \mathrm{L}$; lysate from $2.5 \times 10^{8}$ platelets). The femoral defects in the second group of rabbits were filled either with $250 \mathrm{~mm}^{3}$ BCP mixed with $250 \mu \mathrm{L}$ of physiological saline (control) or with BCP mixed with $250 \mu \mathrm{L}$ APL. Defect fillers were prepared and used under sterile conditions at time of animal surgery. All animals were sacrificed 6 weeks after implantation.

\subsection{Surgical Procedures}

2.3.1. Anesthesia. Prior to surgery, the rabbits were anesthetized using an intramuscular injection of $0.5 \mathrm{mg} / \mathrm{kg}$ Diazepam (Valium; Roche; Basel, Switzerland), $0.25 \mathrm{mg} / \mathrm{kg}$ metedomidine hydrochloride (Domitor, Virbac; France), and $100 \mathrm{mg} / \mathrm{kg}$ Ketamine hydrochloride (Ketalar 500, Pfizer; France). The anesthetized animals were prepared for aseptic surgery, shaved, and disinfected; both lower limb sites were draped using standard sterile procedures.

2.3.2. Surgical Procedure. The surgical technique used was adapted from the one described by Soffer et al. [18]. Briefly, a longitudinal skin incision was made to expose the distal lateral aspect of each femoral condyle. A circular cortical window was formed using a $6 \mathrm{~mm}$ wide internal diameter trephine. A cylindrical critical-size defect of $10 \mathrm{~mm}$ deep and $5.5 \mathrm{~mm}$ wide was then created in the lateral condyle in a stepwise fashion using color-coded drills, (Diffusion International, Paris, France). These cavities were thoroughly rinsed with isotonic saline to remove bone debris and then, using a specially designed injector and applying a light pressure, they were filled with DBBM or BCP mixed with either physiological saline or APL. The aforementioned fillers were prepared and used under sterile conditions at the time of animal surgery. Each soft tissue wound was closed in two successive layers soft tissue and skin, respectively, using resorbable Vicryl 5/0 sutures, and the surgical site was disinfected.

2.3.3. Postsurgical Animal Treatment. All animals were given intramuscular injections of $0.2 \mathrm{mg} / \mathrm{kg}$ metoxicam (Metakam Boehringer Ingelheim Vetmedica; GmbH Germany) to relieve pain during the postoperative $24 \mathrm{~h}$ period. Prophylactic antibacterial treatment, consisting of $25 \mathrm{mg} / \mathrm{kg}$ animal weight sulfadimethoxine trimethoprim (Copylap Biové; France), was also administrated for 5 days after surgery by qualified animal-care staff. After surgery, all operated animals were monitored until they were able to walk on their own. The rabbits were sacrificed using an overdose of pentobarbital 6 weeks after implantation. At that time, the femoral condyles were removed and cleared of the surrounding soft tissue. All excised bone tissue specimens were prepared for histological analysis as described in the histology section.

2.4. Histology. All excised specimens were examined histologically using an established procedure for nondemineralized bone [23]. Each excised bone specimen was cleared of soft tissue, fixed in $10 \%$ phosphate-buffered formalin, rinsed in water, dehydrated in ethanol, cleared in xylene, and embedded in plastic resin (Technovit 7200 VCL, Kulzer \& Co. GmbH, Wehreim, Germany). Radiographs were taken to ensure appropriate defect orientation for subsequent histologic sectioning. The femoral condyles were sectioned perpendicular to the long axis of each defect. Each section was then ground down to a thickness of $100 \mu \mathrm{m}$ using the Exact Grinding System (Exact Aparatebau GmbH Norderstedt, Germany) and polished down to $80 \mu \mathrm{m}$. All tissue specimens were then stained using Giemsa-Paragon for histomorphometric analysis.

2.5. Histomorphometry. Three randomly selected sections per condyle and per animal were analyzed. Each section was examined under a light microscope (Olympus BX 60, Olympus Corporation, Tokyo, Japan) connected to a digital camera (Olympus, E330). New bone formation was quantified using Image Tool 3.0 software (UTHSCA, SanAntonio, TX, USA). The spatial calibration of the histological sections was done by drawing manually the surface of the newly formed bone and the residual bone substitutes. The total surface was obtained by adding all the delimited areas measurements (in $\mathrm{mm}^{2}$ ). The distribution of newly formed bone and residual implanted material was also determined by classifying the defect area into an inner zone (IC) of $2.5 \mathrm{~mm}$ and an outer zone (OC), including the remaining of the $5.5 \mathrm{~mm}$ defect (Figure 1).

2.6. Statistical Analysis. Two-way analysis of variance between groups was conducted to determine the effect of bone substitutes with APL on new bone formation and on the resorption of these materials. Variables were tested for normal distribution and equality of variance using the Kolmogorov Smirnov and Levene tests, respectively. Comparison of the data between the different groups tested was performed using the Student's $t$-test. All statistical analyses were performed using commercially available software programs (SPSS for Windows version 16.0). Significance was defined as a $P$ value of less than 0.05 .

\section{Results}

3.1. Histological Observation. All rabbits recovered within 2-3 hours after surgery. In all cases, tissue healing was uneventful. Rabbits were systematically checked in order to detect any problems during the experimental period and they were weighed every two weeks. No postsurgical complications, 


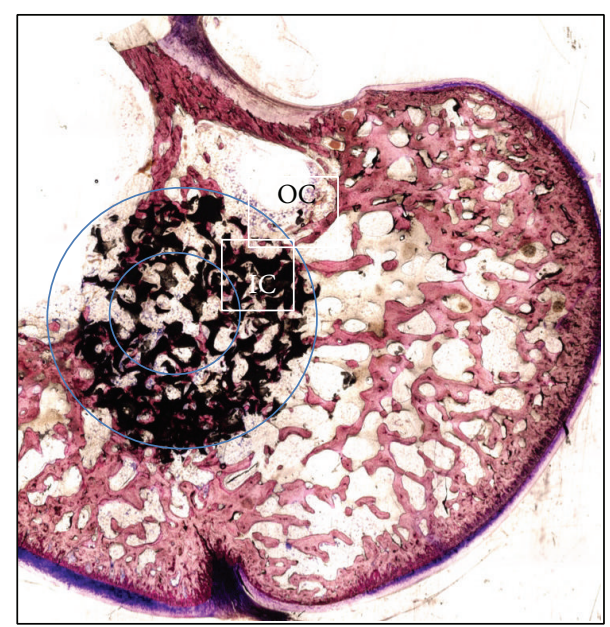

FIGURE 1: Schematic representation of a condyle section. The newly formed bone and the residual material distribution were evaluated separately in the inner (IC) and outer zones (OC) at 6 weeks after implantation.

infections, animal behavior, or general health changes were observed after surgery and for the duration of the study. Rabbits presented a normal weight gain of 103,8 g.

After 6 weeks of implantation, histological analysis of specimens excised from all animals tested showed no inflammatory reactions. New bone formation was evident in close contact to both the BCP and DBBM particles that have been used to fill the femoral defects in the present study, even in the central part of the defect (Figures 2(a) and 3(a)).

In the defects filled with DBBM, newly formed trabecular bone with wide marrow spaces and residual particles were present. The DBBM particles were distributed over the entire grafted area of the femoral defects and were surrounded and bridged by newly formed bone (Figure 2(b)). Neither gaps nor connective fibrous tissues were present at the bone biomaterial interface. A thin layer of osteoid and osteoblast lining-cells was observed in some areas around DBBM particles (Figures 2(b) and 2(c)). Multinucleated giant cells or Howship lacunae, pits, or resorptive trails were not seen on the DBBM surface regardless of the presence or absence of APL. The DBBM thus exhibited osteoconductive properties but not ongoing material resorption.

In the defects filled with BCP, the material particles were distributed over the entire defect area and they were surrounded by newly formed bone (Figure 3(a)) both in the presence and absence of APL addition. Some particles were covered by a layer of newly formed bone (Figure 3(b)). Sites where the material had been resorbed had new bone and marrow spaces. Some lining osteoblasts, as well as multinuclear phagocytic cells, were observed at the surface of the BCP particles (Figures 3(c) and 3(d)). In some areas, particles were embedded in bone; in such cases, osteocytic lacunae were observed. Examination at high magnification $(\times 40)$ revealed that the most newly formed bone had woven bone characteristics. New bone formation was more prominent in the outer circle (OC), bridging with the edges of the defects, than in the inner circle (IC).

3.2. Histomorphometric Results. At 6 weeks after implantation, the bone areas in defects filled with BCP were similar to those observed in defects filled with BCP+APL (6.69 \pm $1.53 \mathrm{~mm}^{2}$ and $6.57 \pm 1.90 \mathrm{~mm}^{2}$, respectively; Figure 4). The amount of bone formed in defects filled with either DBBM or with DBBM+APL was also similar $\left(4.49 \pm 1.19 \mathrm{~mm}^{2}\right.$ and $4.28 \pm 1.42 \mathrm{~mm}^{2}$, resp.). The addition of APL did not promote new bone formation above the extent obtained with the two bone substitutes tested alone. In contrast, the bone areas in defects filled with BCP were significantly $(P<0.0001)$ higher than those observed when DBBM alone was used as the filler. Specifically, the mean difference in the bone amount in defects filled with either BCP or DBBM was $2.29 \mathrm{~mm}^{2}$ (confidence interval (CI) 1.23-3.36) in the absence of APL and $2.19 \mathrm{~mm}^{2}$ (confidence interval (CI) 1.13-3.25) in the presence of APL. For the two substitute materials as well as for all of the experimental conditions tested in the present study, the bone surface areas in the outer zone (OC) were significantly higher than those observed in the inner zone (IC) of the defects (for BCP: $P<0.0001$ in the presence and absence of APL; for DBBM: $P<0.037$ and $P<0.001$ in the presence and absence of APL; Figure 5). Distribution of newly formed bone throughout the respective original defect was not affected by the presence or absence of APL. The bone surface area at the inner as well as at the outer zones of the defects was significantly $(P<0.001)$ higher when the defects were filled with BCP than when they were filled with DBBM (Figure 5).

At 6 weeks after implantation, the surface areas of the residual implanted material in defects filled with BCP was similar to those in defects filled with $\mathrm{BCP}+\mathrm{APL}$ formulations $\left(9.43 \pm 1.89 \mathrm{~mm}^{2}\right.$ and $9.25 \pm 1.61 \mathrm{~mm}^{2}$, respectively; Figure 6). In addition, the surface areas of the residual material in defects filled with DBBM was similar to those filled with $\mathrm{DBBM}+\mathrm{APL}$ formulations $\left(14.61 \pm 1.64 \mathrm{~mm}^{2}\right.$ and $14.36 \pm$ $1.93 \mathrm{~mm}^{2}$, resp.). The amount of implanted material in defects filled with BCP was significantly $(P<0.0001)$ lower (specifically, close to 65\%) compared to that observed with DBBM regardless of the presence of APL. Analysis of the distribution of the nonresorbed implant material throughout the defect provided evidence that, in the defects filled with BCP, the amount of residual material was significantly $(P<$ 0.0001 ) higher in the outer zone than in the inner zone of the defect (mean difference $1.04 \mathrm{~mm}^{2}$; confidence interval (CI): 0.70-0.84; Figure 7). Similar results were observed in the defects filled with $\mathrm{BCP}+\mathrm{APL}$ formulations (mean difference $0.95 \mathrm{~mm}^{2} ; P<0.0001$; confidence interval (CI): 1.11-0.79). In contrast, the amount of residual material present in the defects filled with DBBM in either the presence or the absence of APL was similar in the inner and the outer zones (mean difference $0.06 \mathrm{~mm}^{2} ; P<0.43$; confidence interval (CI): $0.09-0.21$ and $0.07 \mathrm{~mm}^{2} ; P<0.368$; confidence interval (CI): $0.08-0.22$ with and without APL, resp.). 


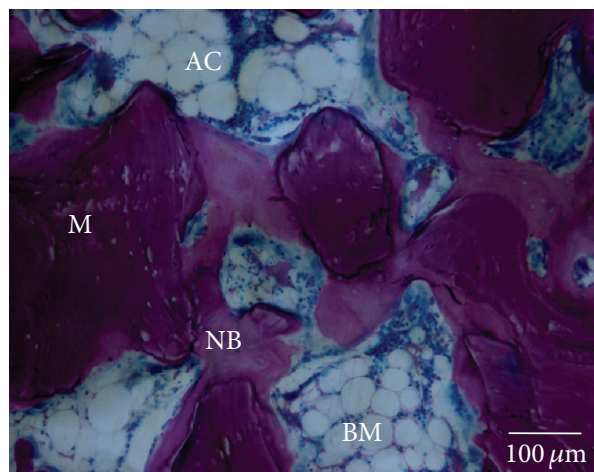

(a)

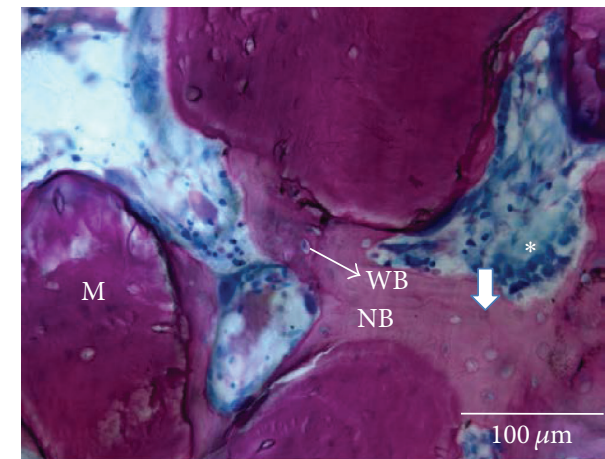

(b)

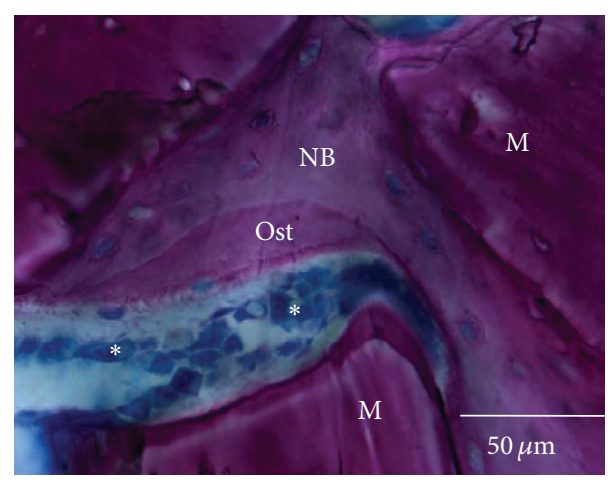

(c)

FIGURE 2: Representative light micrographs illustrating histological details of a defect grafted with DBBM at 6 weeks after implantation. $\operatorname{DBBM}(\mathrm{M})$ surrounded and bridged by newly formed bone (NB). The increased number of adipocytes (AC) in the wide marrow spaces (BM) indicates bone marrow maturation $((\mathrm{a})$; magnification $\times 10)$. Woven bone $(\mathrm{WB})$ and mature paralleled-fiber bone (arrows) were observed ((b); magnification $\times 20)$. Osteoblast-lining cells (asterisks) on the osteoid surface $(\mathrm{OST})$ indicate ongoing bone formation ((b); magnification $\times 20$ and $((c)$; magnification $\times 40)$. Stain: Giemsa-Paragon.

\section{Discussion}

This study compared the osteoconductive and resorption behavior of BCP and DBBM alone or associated with APL, in critical-size rabbit femoral defects. Rabbit is a validated model, currently used in bone reconstruction research. Its size and development level allow performing easy surgical procedures [34]. In particular, the femoral site is an interesting site for the evaluation of the biofunctionality of biomaterials because of the equilibrium of the osteogenesis and the biodegradation activities [35]. In order to achieve standardization in the evaluation of bone substitutes, different authors have developed a critical-size defect model. A critical-size defect is defined as the smallest intraosseous wound that does not heal by bone formation during the lifetime of the animal [36, 37]. According to international standards (ISO 10993-6), bone substitute materials must be evaluated in bone defects of at least $2 \mathrm{~mm}$ diameter and $6 \mathrm{~mm}$ in length for rabbit femurs. DBBM and BCP are the most widely used grafting materials in oral, maxillofacial, and implant surgery [1, 7] and they are frequently used associated with platelet concentrates $[14,24]$. However, there are few experimental studies in the literature focusing on the evaluation of their regenerative potential using standardized intrabony defects within the same study [11] and, to our knowledge, none has used critical-size femoral defects to compare the regenerative properties of these bone substitutes.

Results obtained in this study showed the presence of newly formed bone in close contact with the particles of DBBM and BCP as reported in previous preclinical $[9,12,13]$ and clinical studies $[2-4,10]$. Regarding the distribution of newly formed bone, between the inner and outer zones, new bone formation was more prominent in the $\mathrm{OC}$ than in the IC, which shows a slight tendency of augmented bone formation in the outer zone of the defect. Other histomorphometric studies have suggested that the majority of the bone formation starts from the borders of the defect, as a centripetal bone colonization [38]. Interestingly, under the experimental conditions of the present study, significantly $(P<0.001)$ more bone formation was observed in the BCP group (close to $70 \%$ ) after 6 weeks implantation time in the presence or absence of additional APL (Figure 4). A similar tendency was described in standardized intrabony defects when BCP was compared to pure hydroxyapatite in the mandible of minipigs at 4 and 8 weeks after implantation [9]. In contrast to the results of the present study, BCP and DBBM rendered similar amount of newly formed bone in noncritical size intrabony defects in the mandible of minipigs at 4 and 8 


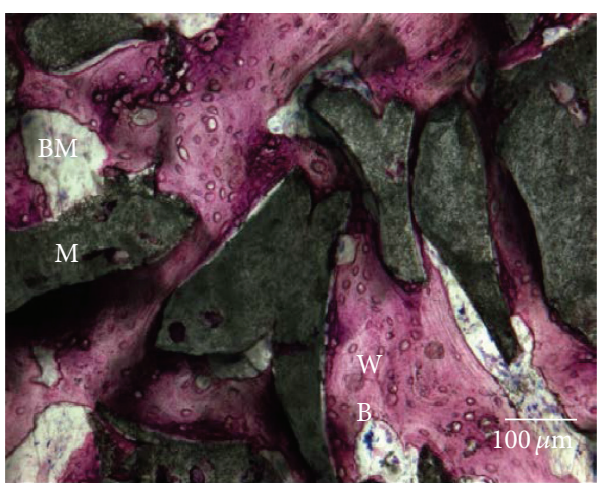

(a)

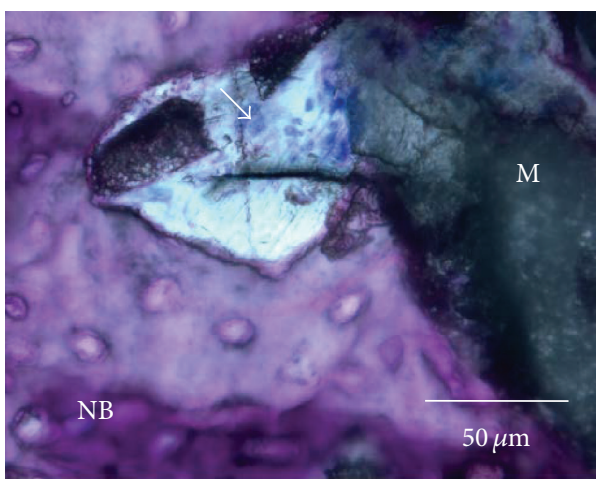

(c)

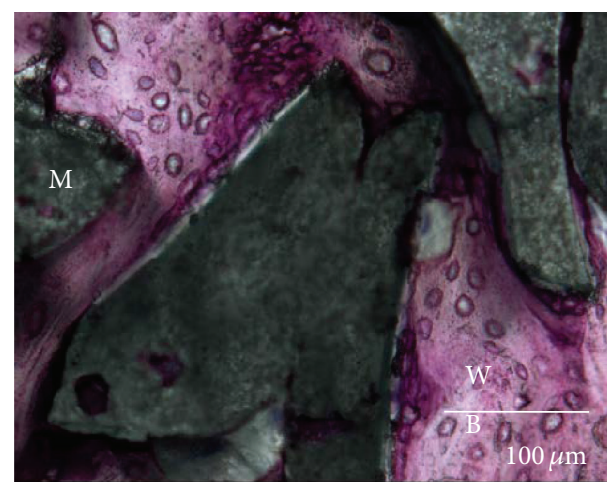

(b)

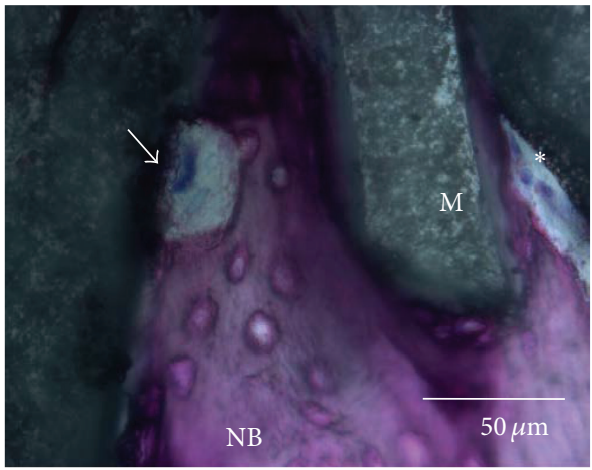

(d)

FIGURE 3: Representative light micrographs illustrating histological details of a defect grafted with BCP at 6 weeks after implantation. BCP surrounded and bridged by newly formed bone ( $(\mathrm{a})$; magnification $\times 10$ and (b); magnification $\times 20)$ ). Mostly newly formed bone had wovenbone (WB) characteristics (b); giant multinuclear cells (arrows) were observed on the BCP particles ((c) and Frame (d); magnification $\times 40)$. Stain: Giemsa-Paragon.

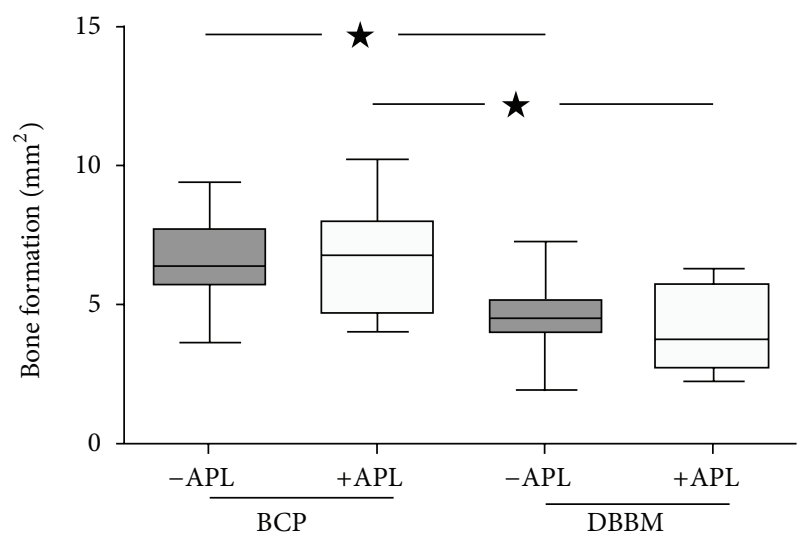

FIGURE 4: Box plot showing bone formation area in the defect at 6 weeks after implantation. In the defects filled with BCP, the newly formed bone area was significantly higher than that observed in the defects filled with DBBM $\left({ }^{*} P<0.001\right)$. No significant differences were observed between defects filled with BCP and BCP+APL, as well as between defects filled with DBBM and DBBM+APL.

weeks after implantation [11], in the rabbit calvaria [12] as well as around implant placed in circumferential defects in dogs mandibles at 8 weeks after implantation [13]. The reasons for these differences are not clear; however, the anatomical and dimensional characteristics of the defects sites (femur in the present study versus calvaria) [12] or mandible [11] and the duration of the study ( 6 weeks in the present study versus 4,8 , and 16 weeks in others) $[11,12])$ may be contributory factors. The efficiency of BCP could be related to its partial dissolution and the enhanced bioactivity of soluble TCP [39]. Further studies with different healing intervals are necessary to clarify the differences between DBBM and BCP osteoconduction efficiency as well as the characteristics of the newly formed bone in their presence.

The most striking difference between the two bone substitutes tested in the present study was their resorption behavior. For the same volume of implanted material, the amount of BCP residual particles in the defect was significantly $(P<$ 0.001 ) lower (specifically, close to 65\%) than that observed in defects filled with DBBM at 6 weeks after implantation. In contrast, a limited resorption was observed in mandible bone defects in minipigs [11] and no BCP resorption in calvaria bone defects in rabbits at 4 and even at 16 weeks after implantation [12]. This disparity may be explained by the differences in the anatomical site of the defect; calvaria is a site where blood supply and bone marrow are poor. However, the results of this work are in accordance with previous studies that reported also approximately 50\% resorption of 


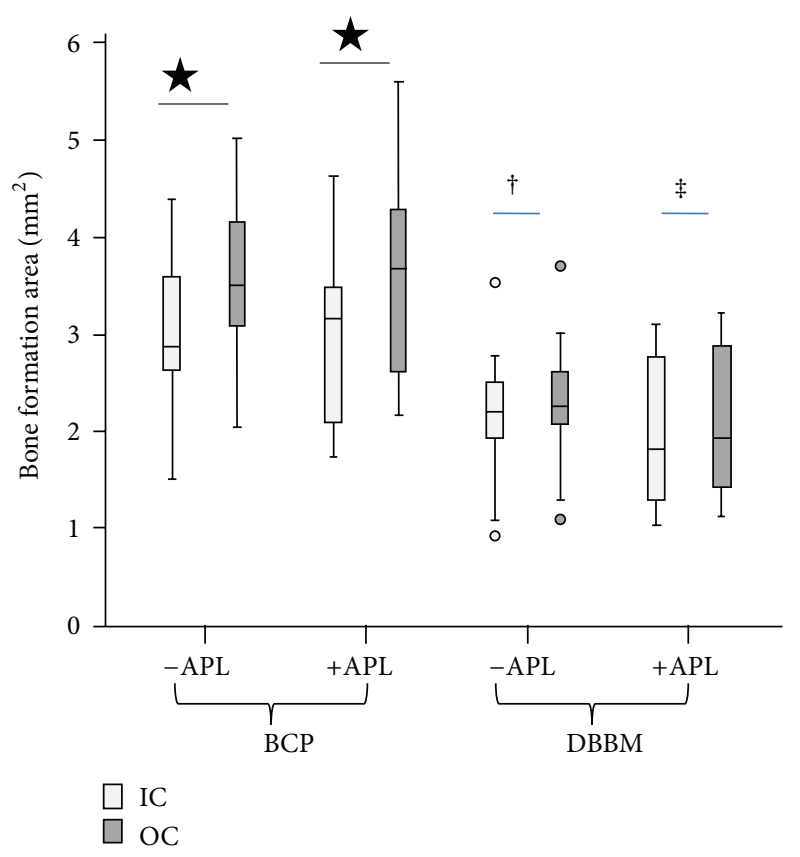

FIGURE 5: Box plot showing bone formation distribution throughout the defect at 6 weeks after implantation. For the two filling materials, the bone surface areas in the outer zone (OC) were significantly higher than those observed in the inner zone (IC) of the defects (for $\mathrm{BCP}^{*} P<0.0001$ in the presence and absence of APL; for $\mathrm{DBBM}^{\dagger} P$ $<0.001$ and ${ }^{\ddagger} P<0.037$ in the absence and presence of APL).

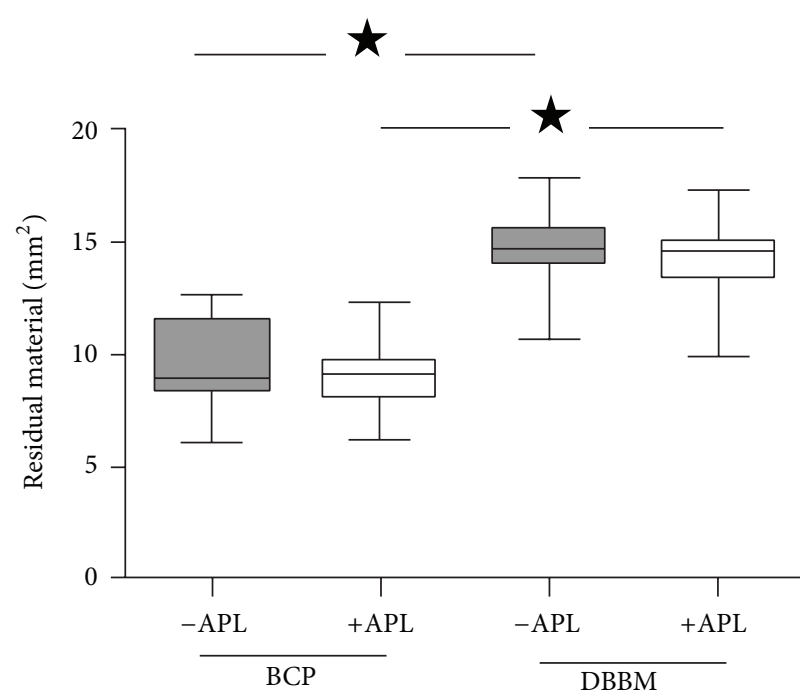

FIGURE 6: Box plot showing residual material area in the defect at 6 weeks after implantation. In the defects filled with DBBM, the amount of residual material was significantly $\left({ }^{*} P<0.0001\right)$ higher than that observed in the defects filled with BCP.

BCP (60/40 HA/TCP ratio) in osseous defects in rabbits within one year after implantation [40]. The resorption of implanted BCP particles provided space and may explain the higher new bone formation observed with this material. Comparison of the distribution of the nonresorbed material throughout the defect showed that, in the defects filled with

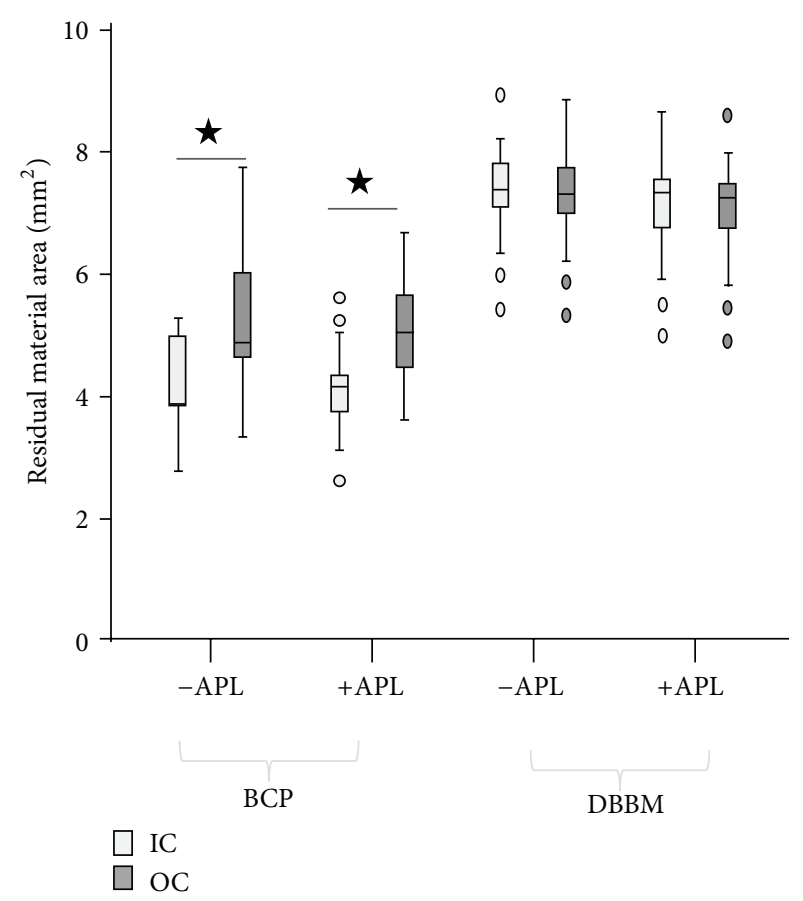

FIGURE 7: Box plot showing residual material distribution throughout the defect. In the defects filled with BCP, the amount of residual material was significantly $\left({ }^{*} P<0.0001\right)$ higher in the outer zone (OC) than in the inner zone (IC) of the defect. In contrast, the amount of residual material present in the defects filled with DBBM was similar in the inner and the outer zones.

BCP, the amount of the residual material was significantly $(P<0.001)$ higher in the outer zone than in the inner zone of the defect. This finding may be correlated to the greater bone formation observed around BCP particles in continuity to the surrounding bone. Regarding the DBBM resorption, residual material in defects filled with DBBM was significantly $(P<0.001)$ higher than that observed in defects filled with $\mathrm{BCP}$, indicating that $\mathrm{DBBM}$ resorption is very slow [7]. Moreover, under the experimental conditions of the present study, multinucleated cells were not observed on the DBBM particles in line with previous findings [6].

Since the use of high local concentrations of plateletderived soluble compounds with bone substitute material remains an attractive strategy for improving bone repair given its autologous origin, minimally invasive nature, and costeffective ratio, but that it has not provided consistent results, the present study was motivated to explore bone healing in defects filled with either DBBM or BCP in the presence of APL. The results obtained at 6 weeks after implantation in the rabbit femoral defects showed that osteoconduction of BCP particles was more pronounced than in the case of DBBM, and the addition of PL did not affect those outcomes. A direct comparison between the results of the present study and published reports on platelet-derived bioactive components is difficult because of the differences in the platelet preparation (PRP, PRF, and APL) and/or in the study design [26]. Nevertheless, the findings of the present study corroborate those of other animal studies, which also showed 
that PRP in conjunction with DBBM in critical-size rabbit calvaria [21] and frontal defect of goat $[17,20]$ does not improve DBBM osteoconductivity. As regards BCP, PRP in combination with BCP (ratio 60/40) was tested only in rat calvaria defects $[19,23]$ and no positive effect of PRP was established. However, recently it has been described that PL coating of BCP increases in vitro chemoattraction and adhesion of MSCs and improves in vivo neovascularization and new bone formation [33].

Moreover, it has been suggested that platelet concentration plays a role in the effect of platelet concentrates in bone healing. In the present study, APL with a platelet concentration of $10^{6}$ platelets $/ \mu \mathrm{L}$ (3.5 to $5 \mathrm{X}$ fold of baseline) was used. With a similar concentration, previous studies have shown biological effects using rabbit PL [28] or rabbit PRP [41] APL with a concentration of $10^{6}$ platelets resulting in higher resorption of calcium carbonate ceramic in rabbit femoral defects [18]. PRP with platelet concentration ranging from $9.5 \times 10^{5}$ to $1.7 \times 10^{6}$ platelets $/ \mu \mathrm{L}$ showed improved peri-implant bone regeneration in rabbits [41]. Below this range the effect is suboptimal; beyond this range, there may be a parodoxical inhibitory effect. The time period during which platelet growth factors remain active [42] and the bioavailability of platelet components in relation to DBBM and/or BCP dissolution in osseous defect could explain the absence of platelet concentrates effect on tissue repair. These hypotheses were not explored in this work, and further investigations may be needed to optimize the use of platelet concentrates. It is not clear how far the results of this rabbit study can be extrapolated to different species. Nevertheless, previous studies reported biological effects using autologous rabbit as well as miniature pig, dog, and human PL.

Moreover, the quantitative histomorphometric measurements performed in this study demonstrated that the addition of APL did not affect the resorption of either DBBM or BCP. However, previous studies reported that platelet concentrates increase the resorption of carbonate phosphate [18] and carbonated apatite calcium phosphate particles [43] probably by triggering anti-inflammatory reaction and/or biological responses in the early events of healing [44]. In contrast to DBBM, that did not or slowly resorbed, and BCP that resorbed mildly, calcium carbonate is a material with high and fast resorption. The discrepancy observed in the resorption pattern of these materials, related to the material surface properties and the $\mathrm{PH}$ of the biological environment, could influence the adsorption and the release of PL adsorbed proteins and growth factors.

In summary, this study demonstrated the osteoconductive properties of these two biomaterials in criticalsize osseous defects and showed in a quantitative way that compared to DBBM, BCP was resorbed and promoted a greater bone formation while APL addition had no impact on those outcomes. Results obtained in the present investigation are, therefore, relevant to the contribution of BCP and DBBM particles, used either alone or in conjunction with autologous bioactive compounds released from platelet concentrates to promote intrabony defects repair.

\section{Conflict of Interests}

The authors report no conflict of interests related to this study.

\section{Acknowledgments}

The authors gratefully acknowledge the help of Professor Rena Bizios for the critical review of the paper. The authors wish to thank Mr. Charbel Mansour for the technical assistance in the preparation of the histological sections.

\section{References}

[1] M. Hallman and A. Thor, "Bone substitutes and growth factors as an alternative/complement to autogenous bone for grafting in implant dentistry," Periodontology 2000, vol. 47, no. 1, pp. 172192, 2008.

[2] N. Mardas, V. Chadha, and N. Donos, "Alveolar ridge preservation with guided bone regeneration and a synthetic bone substitute or a bovine-derived xenograft: a randomized, controlled clinical trial," Clinical Oral Implants Research, vol. 21, no. 7, pp. 688-698, 2010.

[3] C. Lindgren, A. Mordenfeld, and M. Hallman, "A prospective 1-year clinical and radiographic study of implants placed after maxillary sinus floor augmentation with synthetic biphasic calcium phosphate or deproteinized bovine bone," Clinical Implant Dentistry and Related Research, vol. 14, no. 1, pp. 41-50, 2012.

[4] C. M. Schmitt, H. Doering, T. Schmidt, R. Lutz, F. W. Neukam, and K. A. Schlegel, "Histological results after maxillary sinus augmentation with Straumann $\AA$ BoneCeramic, Bio-Oss $®$, Puros $\AA$, and autologous bone. A randomized controlled clinical trial," Clinical Oral Implants Research, vol. 24, no. 5, pp. 576585, 2013.

[5] V. Benezra Rosen, L. W. Hobbs, and M. Spector, "The ultrastructure of anorganic bovine bone and selected synthetic hyroxyapatites used as bone graft substitute materials," Biomaterials, vol. 23, no. 3, pp. 921-928, 2002.

[6] M. G. Araújo, D. Carmagnola, T. Berglundh, B. Thilander, and J. Lindhe, "Orthodontic movement in bone defects augmented with Bio-Oss®. An experimental study in dogs," Journal of Clinical Periodontology, vol. 28, no. 1, pp. 73-80, 2001.

[7] N. Baldini, M. De Sanctis, and M. Ferrari, "Deproteinized bovine bone in periodontal and implant surgery," Dental Materials, vol. 27, no. 1, pp. 61-70, 2011.

[8] C. Schopper, F. Ziya-Ghazvini, W. Goriwoda et al., "HA/TCP compounding of a porous $\mathrm{CaP}$ biomaterial improves bone formation and scaffold degradation-a long-term histological study," Journal of Biomedical Materials Research B, Applied Biomaterials, vol. 74, no. 1, pp. 458-467, 2005.

[9] S. S. Jensen, A. Yeo, M. Dard, E. Hunziker, R. Schenk, and D. Buser, "Evaluation of a novel biphasic calcium phosphate in standardized bone defects. A histologic and histomorphometric study in the mandibles of minipigs," Clinical Oral Implants Research, vol. 18, no. 6, pp. 752-760, 2007.

[10] L. Cordaro, D. D. Bosshardt, P. Palattella, W. Rao, G. Serino, and M. Chiapasco, "Maxillary sinus grafting with Bio-Oss $®$ or Straumann $₫$ Bone Ceramic: histomorphometric results from a randomized controlled multicenter clinical trial," Clinical Oral Implants Research, vol. 19, no. 8, pp. 796-803, 2008.

[11] S. S. Jensen, M. M. Bornstein, M. Dard, D. D. Bosshardt, and D. Buser, "Comparative study of biphasic calcium phosphates 
with different HA/TCP ratios in mandibular bone defects. A long-term histomorphometric study in minipigs," Journal of Biomedical Materials Research B, Applied Biomaterials, vol. 90, pp. 171-181, 2009.

[12] A. R. Rokn, M. A. Khodadoostan, G. A. A. R. Rasouli et al., "Bone formation with two types of grafting materials: a histologic and histomorphometric study," Open Dentistry Journal, vol. 5, no. 1, pp. 96-104, 2011.

[13] A. A. Antunes, P. Oliveira Neto, E. de Santis, M. Caneva, D. Botticelli, and L. A. Salata, "Comparisons between Bio-Oss $\AA$

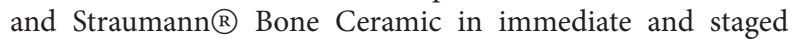
implant placement in dogs mandible bone defects," Clinical Oral Implants Research, vol. 24, no. 2, pp. 135-142, 2013.

[14] M. Del Fabbro, M. Bortolin, S. Taschieri, and R. Weinstein, "Is platelet concentrate advantageous for the surgical treatment of periodontal diseases? A systematic review and meta-analysis," Journal of Periodontology, vol. 82, no. 8, pp. 1100-1111, 2011.

[15] A. Simonpieri, M. Del Corso, A. Vervelle et al., "Current knowledge and perspectives for the use of Platelet-Rich Plasma (PRP) and Platelet-Rich Fibrin (PRF) in oral and maxillofacial surgery part 2: bone graft, implant and reconstructive surgery," Current Pharmaceutical Biotechnology, vol. 13, no. 7, pp. 12311256, 2012.

[16] J. Alsousou, A. Ali, K. Willett, and P. Harrison, "The role of platelet-rich plasma in tissue regeneration," Platelets, vol. 24, no. 3, pp. 173-182, 2013.

[17] J. P. M. Fennis, P. J. W. Stoelinga, and J. A. Jansen, "Mandibular reconstruction: a histological and histomorphometric study on the use of autogenous scaffolds, particulate cortico-cancellous bone grafts and platelet rich plasma in goats," International Journal of Oral and Maxillofacial Surgery, vol. 33, no. 1, pp. 4855, 2004.

[18] E. Soffer, J. P. Ouhayoun, A. Meunier, and F. Anagnostou, "Effects of autologous platelet lysates on ceramic particle resorption and new bone formation in critical size defects: the role of anatomical sites," Journal of Biomedical Materials Research B, Applied Biomaterials, vol. 79, no. 1, pp. 86-94, 2006.

[19] A. S. Plachokova, J. Van Den Dolder, P. J. Stoelinga, and J. A. Jansen, "The bone regenerative effect of platelet-rich plasma in combination with an osteoconductive material in rat cranial defects," Clinical Oral Implants Research, vol. 17, no. 3, pp. 305311, 2006.

[20] R. E. C. M. Mooren, A. C. A. Dankers, M. A. W. Merkx, E. M. Bronkhorst, J. A. Jansen, and P. J. W. Stoelinga, "The effect of platelet-rich plasma on early and late bone healing using a mixture of particulate autogenous cancellous bone and BioOss®: an experimental study in goats," International Journal of Oral and Maxillofacial Surgery, vol. 39, no. 4, pp. 371-378, 2010.

[21] F. S. Rocha, L. M. A. Ramos, J. D. Batista, D. Zanetta-Barbosa, E. A. V. Ferro, and P. Dechichi, "Bovine anorganic bone graft associated with platelet-rich plasma: histologic analysis in rabbit calvaria," Journal of Oral Implantology, vol. 37, no. 5, pp. 511-518, 2011.

[22] M. Q. Kurikchy, N. H. Al-Rawi, R. S. Ayoub, and S. S. Mohammed, "Histological evaluation of bone healing using organic bovine bone in combination with platelet-rich plasma (an experimental study on rabbits)," Clinical Oral Investigations, vol. 17, no. 3, pp. 897-904, 2013.

[23] G. Faratzis, M. Leventis, E. Chrysomali et al., "Effect of autologous platelet-rich plasma in combination with a biphasic synthetic graft material on bone healing in critical-size cranial defects," Journal of Craniofacial Surgery, vol. 23, no. 5, pp. 13181323, 2012.

[24] H. Schliephake, "Clinical efficacy of growth factors to enhance tissue repair in oral and maxillofacial reconstruction: a systematic review," Clinical Implant Dentistry and Related Research, 2013.

[25] P. Kasten, J. Vogel, F. Geiger, P. Niemeyer, R. Luginbühl, and K. Szalay, "The effect of platelet-rich plasma on healing in criticalsize long-bone defects," Biomaterials, vol. 29, no. 29, pp. 39833992, 2008.

[26] A. D. Mazzocca, M. B. R. McCarthy, D. M. Chowaniec et al., "Platelet-rich plasma differs according to preparation method and human variability," Journal of Bone and Joint Surgery A, vol. 94, no. 4, pp. 308-316, 2012.

[27] C. Doucet, I. Ernou, Y. Zhang et al., "Platelet lysates promote mesenchymal stem cell expansion: a safety substitute for animal serum in cell-based therapy applications," Journal of Cellular Physiology, vol. 205, no. 2, pp. 228-236, 2005.

[28] E. Soffer, J.-P. Ouhayoun, C. Dosquet, A. Meunier, and F. Anagnostou, "Effects of platelet lysates on select bone cell functions," Clinical Oral Implants Research, vol. 15, no. 5, pp. 581-588, 2004.

[29] N. Chevallier, F. Anagnostou, S. Zilber et al., "Osteoblastic differentiation of human mesenchymal stem cells with platelet lysate," Biomaterials, vol. 31, no. 2, pp. 270-278, 2010.

[30] R. Crespo-Diaz, A. Behfar, G. W. Butler et al., "Platelet lysate consisting of a natural repair proteome supports human mesenchymal stem cell proliferation and chromosomal stability," Cell Transplantation, vol. 20, no. 6, pp. 797-811, 2011.

[31] C. M. Caramella, G. Sandri, S. Rossi et al., "New therapeutic platforms for the treatment of epithelial and cutaneous lesions," Current Drug Delivery, vol. 10, no. 1, pp. 18-31, 2013.

[32] B. Dozza, C. Di Bella, E. Lucarelli et al., "Mesenchymal stem cells and platelet lysate in fibrin or collagen scaffold promote noncemented hip prosthesis integration," Journal of Orthopaedic Research, vol. 29, no. 6, pp. 961-968, 2011.

[33] J. Leotot, L. Coquelin, G. Bodivit et al., "Platelet lysate coating on scaffolds directly and indirectly enhances cell migration, improving bone and blood vessel formation," Acta Biomaterialia, vol. 9, no. 5, pp. 6630-6640, 2013.

[34] S. Stübinger and M. Dard, "The rabbit as experimental model for research in implant dentistry and related tissue regeneration," Journal of Investigative Surgery, vol. 26, no. 5, pp. 266-282, 2013.

[35] J. X. Lu, A. Gallur, B. Flautre et al., "Comparative study of tissue reactions to calcium phosphate ceramics among cancellous, cortical, and medullar bone sites in rabbits," Journal of Biomedical Materials Research A, vol. 42, no. 3, pp. 357-367, 1998.

[36] J. P. Schmitz and J. O. Hollinger, "The critical size defect as an experimental model for craniomandibulofacial nonunions," Clinical Orthopaedics and Related Research, vol. 205, pp. 299308, 1986.

[37] A. K. Gosain, L. Song, P. Yu et al., "Osteogenesis in cranial defects: reassessment of the concept of critical size and the expression of TGF- $\beta$ isoforms," Plastic and Reconstructive Surgery, vol. 106, no. 2, pp. 360-371, 2000.

[38] N. Miño-Fariña, F. Muñoz-Guzón, M. López-Peña et al., "Quantitative analysis of the resorption and osteoconduction of a macroporous calcium phosphate bone cement for the repair of a critical size defect in the femoral condyle," Veterinary Journal, vol. 179, no. 2, pp. 264-272, 2009. 
[39] G. Daculsi, P. Weiss, J.-M. Bouler, O. Gauthier, F. Millot, and E. Aguado, "Biphasic calcium phosphate/hydrosoluble polymer composites: a new concept for bone and dental substitution biomaterials," Bone, vol. 25, no. 1, pp. 59S-61S, 1999.

[40] L. Le Guehennec, E. Goyenvalle, E. Aguado et al., "Small-animal models for testing macroporous ceramic bone substitutes," Journal of Biomedical Materials Research B, Applied Biomaterials, vol. 72, no. 1, pp. 69-78, 2005.

[41] G. Weibrich, T. Hansen, W. Kleis, R. Buch, and W. E. Hitzler, "Effect of platelet concentration in platelet-rich plasma on periimplant bone regeneration," Bone, vol. 34, no. 4, pp. 665-671, 2004.

[42] R. C. Tsay, J. Vo, A. Burke, S. B. Eisig, H. H. Lu, and R. Landesberg, "Differential growth factor retention by platelet-rich plasma composites," Journal of Oral and Maxillofacial Surgery, vol. 63, no. 4, pp. 521-528, 2005.

[43] P. Jungbluth, M. Wild, J.-P. Grassmann et al., "Platelet-rich plasma on calcium phosphate granules promotes metaphyseal bone healing in mini-pigs," Journal of Orthopaedic Research, vol. 28, no. 11, pp. 1448-1455, 2010.

[44] R. E. Backly, V. Ulivi, L. Tonachini, R. Cancedda, F. Descalzi, and M. Mastrogiacomo, "Platelet lysate induces in vitro wound healing of human keratinocytes associated with a strong proinflammatory response," Tissue Engineering A, vol. 17, no. 13-14, pp. 1787-1800, 2011. 

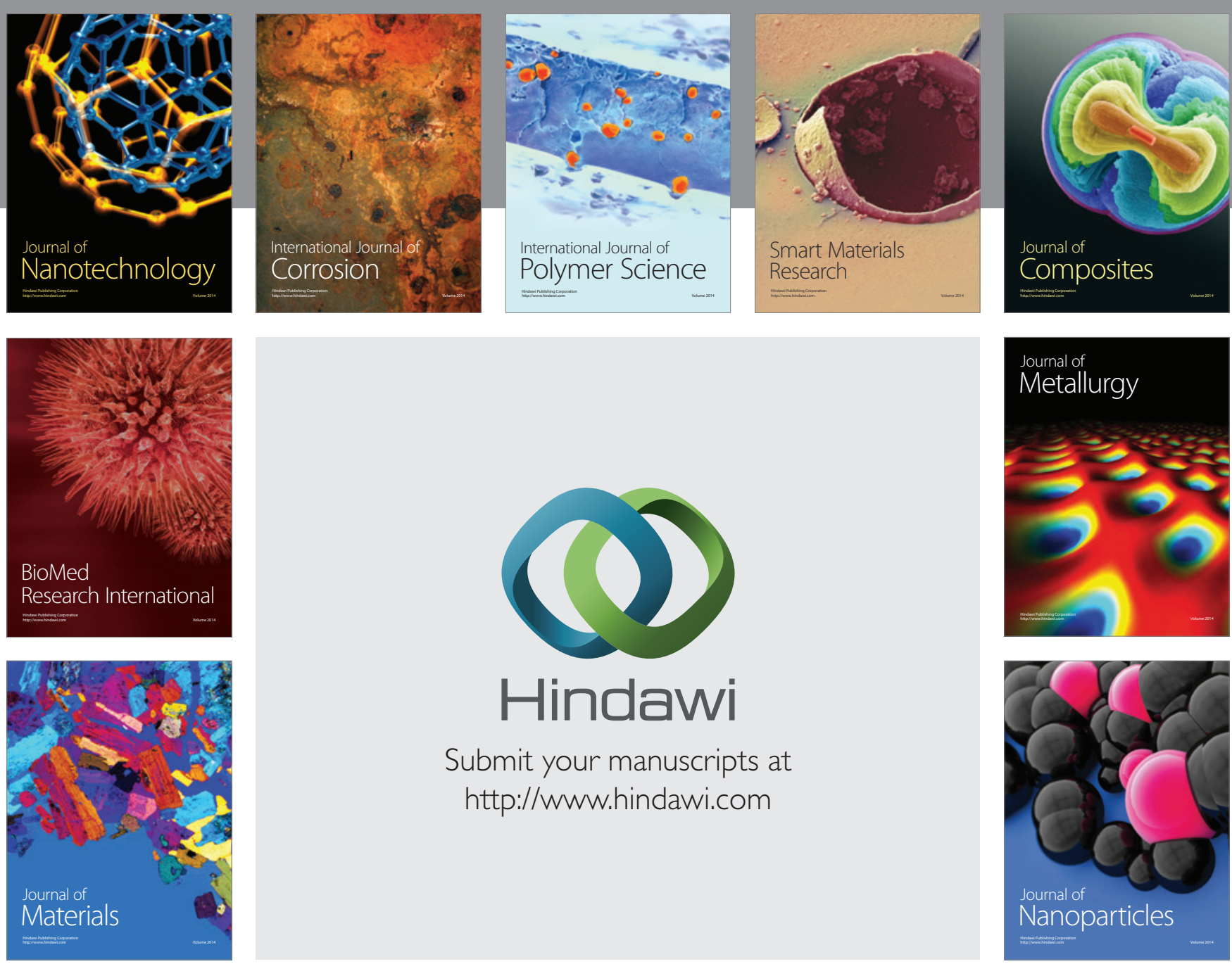

Submit your manuscripts at http://www.hindawi.com
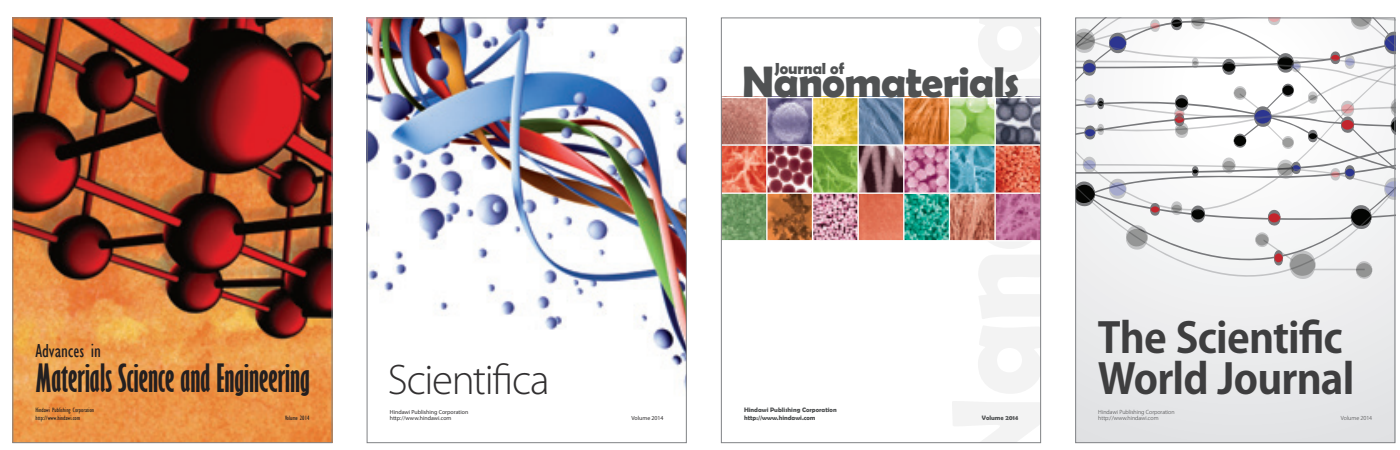

\section{The Scientific World Journal}
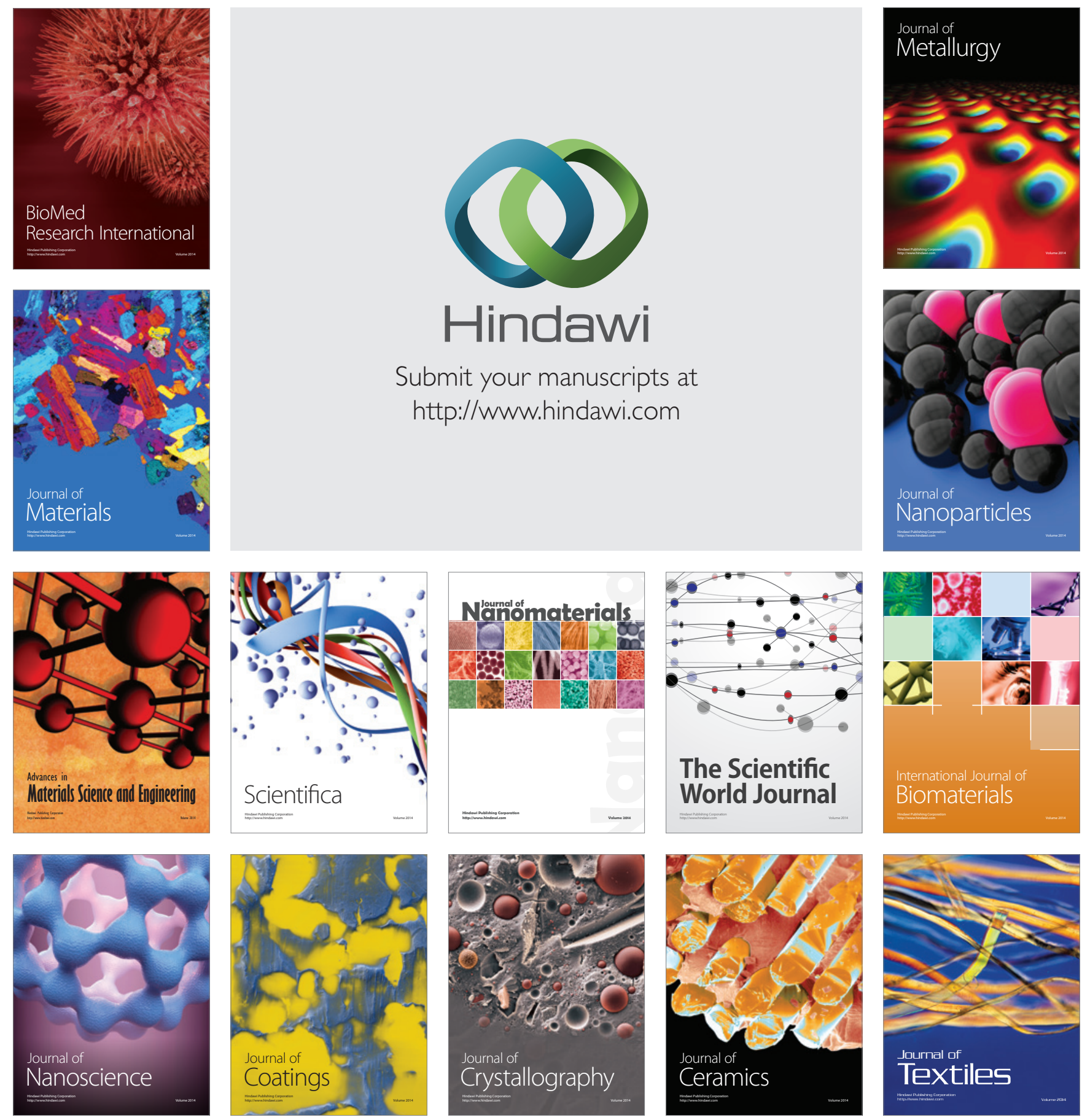